\title{
Die Frauenbilder im Roman ,Die Brücke vom Goldenen Horn“ von der Migrantenliteraturautorin Emine Sevgi Özdamar
}

\author{
Behiye Arabacıoğlu (D), Eskişehir - Şengül Balkaya (D), Eskişehir \\ https://dx.doi.org/10.37583/diyalog. 759418
}

\begin{abstract}
Deutsch)
Die Literatur spiegelt in Werken die Spuren der Kultur, der sie zugehört, wider und ist beauftragt der Gesellschaft Richtung zu geben. Deshalb zeigen literarische Werke die geschichtliche, soziale, wirtschaftliche Seite und Kenntnisse vor dem Hintergrund ihrer Zeit. Durch Migrationserfahrungen wird literarischen Genres ein neues literarisches Genre hinzugefügt, nämlich, die Migrantenliteratur'. Obwohl die Migrantenliteratur andere politisch-historische Hintergründe in sich trägt, kommt es in ihrem Kontext zu ähnlichen Bildern und Problemstellungen des Eigenen und des Fremden. Die Zahl der Schriftstellerinnen in diesem Genre der Literatur ist gering, aber Emin Sevgi Özdamar ist eine weltweit berühmte Schriftstellerin. Özdamars Erfolg ist darin zu sehen, dass sie Wörter, Sprüche und Redewendungen aus ihrer Muttersprache unmittelbar ins Deutsche überträgt. Das Erzählverfahren von Özdamar ist von interkulturellen Bezügen hinsichtlich der deutschen und der türkischen Kultur geprägt. Genau aus diesen Gründen sollen in der vorliegenden Studie die Sichtweise von Frauen, Frauenbildern, verschiedenen weiblichen Rollen in der deutschen und türkischen Gesellschaft im Roman ,Die Brücke vom goldenen Horn von Emine Sevgi Özdamar, einer weltweit bekannten Autorin, analysiert werden. Es wird beabsichtigt, aus dem Blickwinkel einer Schriftstellerin aufzuzeigen, wie sich die Frauenbilder in den Jahren 1966-1975 in Deutschland und in der Türkei darstellten, ob es Unterschiede zwischen den zwei Kulturen gab und vor welchem geschichtlichen und sozialen Hintergrund die Frau, die im Mittelpunkt steht, lebte.
\end{abstract}

Schlüsselwörter: Frauenbilder, Migrantenliteratur, türkische und deutsche Kultur, ,die Brücke vom Goldenen Horn', Emine Sevgi Özdamar

\section{Abstract (English)}

The Images of Women in the Novel 'The Bridge of the Golden Horn' by the Migrant Literature Author Emine Sevgi Özdamar

Through its works, literature reflects the traces of the culture to which they belong and have been nominated to direct society. That is why, literary works show the historical, social, and economic side, and the knowledge about the background of era. Migration experiences add a new literary genre to literary types, such as 'the migrant literature'. Although migrant literature preserves other political and historical backgrounds, similar images and problems of its own and the foreign ones arise in the context. The number of female writers in this genre of literature is quite low but Emine Sevgi Özdamar is one of the well-known worldwide authors. Özdamar's achievement can be observed in the fact that she directly translates words, sayings and idiomatic expressions from her mother tongue into German. Her narrative process is characterized by intercultural references to German and Turkish culture. For these reasons, this study intends to analyze the 
perspectives and images of women, and various female roles in German and Turkish societies mentioned in the novel 'The Bridge of the Golden Horn' by Emine Sevgi Özdamar. Within the scope of this study, it is aimed to demonstrate how the images of women were used to be in Germany and Turkey between the years of 1966-1975; whether there were differences in between two cultures, and how the historical and social situation were presented from a woman writer's point of view.

Keywords: Images of women, migrant literature, Turkish and German culture, The Bridge of the Golden Horn, Emine Sevgi Özdamar

\section{EXTENDED ABSTRACT}

Literature, which is an inseparable part of the society, should be seen as a part of culture because it contains many elements. Literature reflects the traces of the culture to which it belongs to, and also assumes the task of leading the society. In addition to providing information on the historical, social and economic backgrounds of the period in which it is written, it also displays clues about the attitudes and views of society regarding individuals, facts, events and other cultures.

Together with migration, a new literary genre emerged that reflects both the works of the Turkish society to which writers belong, as well as the impressions and images from Germany, where they have acquired a new homeland. The pioneers of migrant literature are those who wanted to express themselves literally among the ones, having immigrated to Germany. Emine Sevgi Özdamar is one of the most interesting authors because she has her own style and plays upon words in her works. She transfers the Turkish words directly into German, which is regarded as quite interesting by the German readers. The most outstanding feature that distinguishes Özdamar from other authors is that she has utilized the expressions, sayings, proverbs, and idioms directly into German. This new style makes her an original writer.

Özdamar, discusses the image of women differently from the first generation writers who reflect immigrant women image as workers immigrating to Germany just to make money. Unlike the previous works, there is the image of a woman who goes to Germany as a worker in order to be a good actress, to study theatre.

In this study, information obtained from the work of a female writer between1966-1975 in both countries Germany and Turkey are presented. It is also investigated whether there are differences between two cultures and historical and social structures remaining in the background. This study aims to investigate images of woman from the perceptive of woman migrant literature writer. So as to reach this aim, the work called 'The Bridge of the Golden Horn' by Emine Sevgi Özdamar is selected. The dates are analyzed with a reader-oriented text analysis approach. The book was read critically by the researchers, and themes related to women's images and roles were determined. In addition, information about the historical and social structure in the background was compiled. In order to contribute to the validity and reliability of the study in accordance with these determined themes and background, the relevant direct quotations were presented. In the analysis of the data, immanent critique was utilized. 
The findings show that intertextuality is at the forefront in this work. Özdamar frequently makes references in her work to the writers and poets she has read and presents sections from the works of these writers and poets. She transfers the historical and social background to the reader from the newspaper headlines and articles she memorizes very well. This provides not only news headlines from Turkey and Germany, also offers news from the world press published at that time. In addition to female images, woman's attributes also play an important role in providing information to the reader with a neutral perspective. Özdamar also provides information to readers like a history book, which enables approaching historical and social facts with a neutral identity.

Özdamar reflects many woman images in her work. One of these woman images is Anatolian women who faces difficult living conditions, gives birth to many children, who is obliged to marry her husband's brother when her husband dies, and is depended on her husband as a result of the patriarchal society. Another woman images are women who feel the pressure of their husband even when they are away from him in Germany, and who are forced to send money to their homeland. In this work, the other women image is the prostitutes that are repressed and seen on the other side of the society living under difficult life conditions.

In this study, the European adoration of the Turkish society in those years, the confidence in the products and goods of Europe are demonstrated to the reader. That's to say, besides being a quite important writer of migrant literature; Özdamar is also a good observer presenting readers the images of women, in other words, female portraits that she draws based on the historical and social facts. 


\section{Einleitung}

$\mathrm{Zu}$ untrennbaren Bestandteilen einer Gesellschaft gehört in erster Reihe ,die Kultur', deren vielfältige Elemente die Gesellschaft selbst gestalten. Diese Elemente beeinflussen sich gegenseitig, indem sie die Kultur prägen und im Rahmen der Maßstäbe der Kultur formuliert werden. Die Literatur spiegelt in Werken die Spuren der Kultur, der sie zugehört, wider und ist beauftragt der Gesellschaft Richtung zu geben. Deshalb zeigen literarische Werke die geschichtliche, soziale, wirtschaftliche Seite und Kenntnisse vor dem Hintergrund der Zeit, in der sie geschrieben wurden, zugleich zeigt die Literatur auch, welches Verhalten sie dem Menschen, den Fakten, Ereignissen und anderen Kulturen und Meinungen gegenüber hatte. Auch wenn die Phänomene in den literarischen Werken aus der Perspektive des Autors behandelt zu werden scheinen, spiegeln die darin enthaltenen Urteile, Vorurteile oder Bilder die Eindrücke der Gesellschaft jener Zeit durch den Autor wider. Insofern kann gesagt werden, dass solche Studien Indikatoren der Geschichte sind, da die Bilder in literarischen Werken Licht auf die soziale Struktur werfen. Während Aksan (vgl. 2005: 2) ,ein Bild‘ als die Übertragung des Dichters, der ein Ereignis, das er beobachtet hat, durch den Filter seines eigenen Geistes widerspiegelt, beschreibt Mutlu (vgl. 2012: 14) jedoch ,das Bild‘ als Grundelement der Möglichkeit, der Poesie einen Sinn zu geben. ,Dem Bild‘ ist nicht nur in der Poesie, sondern zugleich auch in Romanen und in anderen literarischen Genres häufig zu begegnen. Mit der Migration türkischer Arbeiter nach Deutschland wird diesen literarischen Genres ein neues literarisches Genre hinzugefügt, ,die Migrantenliteratur'.

Die Migrantenliteratur in Deutschland unterscheidet sich von anderen literarischen Genres darin, dass sie Spuren der türkischen Kultur trägt und die deutsche Kultur, der sie eigentlich völlig fremd ist, widerspiegelt. Die Migrantenliteratur wird in drei Gruppen eingeteilt (vgl. Öztürk 1999: 75). Als erste Generation wird die Gruppe der ersten Arbeiter in den 60er Jahren genannt, darunter Schriftsteller wie Aras Ören, Bekir Y1ldı, Fakir Baykurt, Güney Dal, Habib Bektas, Nevzat Üstün, Sinasi Dikmen und Yüksel Pazarkaya. Die zweite Generation wird von Schriftstellern wie Alev Tekinay, Aysel Özakın, Feridun Zaimoğlu, Nevfel Cumart, Renan Demirkan, Saliha Scheinhardt, Zafer Şenocak und Zehra Çırak vertreten, die die Kinder der ersten Generation sind, die sich entschieden haben in Deutschland zu bleiben oder zu Bildungszwecken nach Deutschland gegangen sind (vgl. Asutay /Çarıç̧ı 2015: 22). Unter der dritten Generation, die die deutsche und türkische Literatur überschreitet und als Weltliteratur akzeptiert wird, befinden sich Emine Sevgi Özdamar, Kerim Pamuk, Selim Özdoğan und Orkun Ertener (vgl. Balc1 2010: 80). Diese Schriftsteller sind zu Forschungsthemen geworden, es wird viel über sie geschrieben und auch Magisterarbeiten und sogar Dissertationen handeln von ihnen (Kuruyazic1 1996, 1997; Ghaussy 1999; Öztürk 1999; İlk1lıç 2000; Oraliş 2001; Kocadoru 2003; Ekiz 2007; Herzog 2010; Balc1 2010; Can 2011; Er 2013; Uysal- Ünalan 2016; Balc1 /Akgün 2018).

Die Entwicklungen der gesellschaftlichen und sozialen Phänomene haben im Laufe der Jahre auch die Themenbereiche der Migrantenliteratur verändert und geprägt. Das Hauptthema und -problem der ersten Generation begann mit der Migration und war die Anpassung an Deutschland, die dritte Generation dagegen stellte sich in ihren 
Werken als Deutsch mit türkischer Herkunft vor. „Diese neue Generation hat angefangen in ihren Erzählungen Ost-West gemischte Sprachspiele zu verwenden (Can 2011: 143)“, das war der Grund des weltweiten Ruhms. Wie Can feststellt, spiegelt die dritte Generation die Spuren der türkischen Gesellschaft wider, zu der sie gehören und auch ihre Eindrücke und Bilder aus Deutschland, wo sie eine neue Heimat erworben haben, und dies machen sie in einem anderen Stil. Die dritte Generation ist unter anderem auch deshalb anders als die erste, weil sie nicht nur aus männlichen Schriftstellern besteht. In den Werken der ersten Generation ist die weibliche Figur immer als Mutter, Liebhaberin oder Ehefrau anzutreffen, doch in der dritten Generation erscheint die weibliche Figur auch in der Identität des Autors. Obwohl bis heute bereits mehrere Werke über Frauen geschrieben worden sind, ist es von großer Bedeutung, dass Frauen über Frauen schreiben und somit eine Selbstkritik darstellen. Auch in dieser Hinsicht ist die dritte Generation mit der ersten zu vergleichen. Deutlich zu sehen ist jedoch, dass die Zahl der Schriftstellerinnen in diesem Genre der Literatur gering ist. Genau aus diesem Grund soll in der vorliegenden Studie die Sichtweise von Frauen, Frauenbildern, verschiedenen weiblichen Rollen in der deutschen und türkischen Gesellschaft im Roman ,Die Brücke vom goldenen Horn' von Emine Sevgi Özdamar, einer weltweit bekannten Autorin, untersucht werden. Es wird beabsichtigt, aus dem Blickwinkel einer Schriftstellerin aufzuzeigen, wie sich die Frauenbilder in den Jahren 1966-1975 in Deutschland und in der Türkei darstellten, ob es Unterschiede zwischen den zwei Kulturen gab und vor welchem geschichtlichen und sozialen Hintergrund die Frau, die im Mittelpunkt steht, lebte.

\section{Theoretischer Teil}

Die Arbeitslosigkeit und der schwierige Lebensunterhalt in den 60er Jahren in Anatolien brachten die Menschen dazu, in Großstädte und sogar ins Ausland, vor allem nach Deutschland, zu ziehen. Can (2011: 140) definiert die Migration folgendermaßen: „Manchmal aus Notwendigkeit, manchmal aus Anforderungen. Die Migration ist ein politisches Phänomen, das die Fragmentierung von Zeit und Gesellschaft mit der Dominanz seiner historischen politischen Seite verursacht". Diese Definition betont genau die Notwendigkeit der türkischen Gesellschaft und diese Notwendigkeit führt zur Entstehung eines neuen Genres, nämlich zur ,Migrantenliteratur'. Denn die Migration drückt nicht nur einen Ortswechsel aus, sondern rückt zugleich die gesellschaftlichen Interaktionen und kulturellen Übertragungen in den Vordergrund. In den 60er Jahren haben sich Menschen getraut, in Länder zu ziehen, deren Sprache, Kultur, Lebensweisen ihnen fremd waren, nur um besser leben zu können, oder eine Hochzeitsfeier machen zu können, wenn sie wieder zurückkehren. Doch wie auch Çakır (vgl. 2012: 30) betont, haben Türken, die migriert sind, in Deutschland Probleme erlebt. Die Menschen, die in diesen Jahren als Arbeiter migriert sind, zeigen im Allgemeinen ein Türkenbild derjenigen, die Geld verdienen müssen. Im Werk von Özdamar, einer Schriftstellerin der dritten Generation, ist der Fall anders. Dieses Mal ist es nicht eine Person, die Geld verdienen muss, sondern eine Frau, die als Arbeiterin nach Deutschland will, um eine Theaterausbildung machen $\mathrm{zu}$ können und dann 
Theaterschauspielerin werden möchte. Die größte Eigenschaft, die Özdamar von anderen Autoren unterscheidet, ist, dass sie die Ausdrücke, Sprüche, Sprichwörter und Redewendungen aus ihrer Muttersprache direkt ins Deutsche übersetzt und in ihre Werke überträgt. Dieser neue Stil macht sie zu einer originellen Schriftstellerin. Diese Aussagen, die den Deutschen fremd sind, sind für Türken, die Deutsch sprechen, leicht verständlich. Der neue Stil von Özdamar, der mit diesen Sprachspielen entstanden ist, wurde von Literaturkritikern akzeptiert und hochgeschätzt. Özdamars Werke sind nicht nur von der deutschen und türkischen Kultur beeinflusst, sondern auch die Werke, die sie in ihrer Kindheit gelesen hat wie Flaubert, Defoe und Dostojewski und dann die, die sie in Deutschland gelesen hat, Kafka, Büchner, Hölderlin, Böll, Joyce, Conrad und Borchert (vgl. Hammond 2016: 4), hatten einen großen Einfluss auf die Schriftstellerin. Im Werk ,Die Brücke vom goldenen Horn' wird auch betont, dass Özdamar Brecht (S. 36, 67), Tschechow (S. 38), Engels (S. 89), Gorki (S. 89), Lenin (S. 245) und Marx (S. 252) liest. Nachdem Özdamar nach Deutschland gegangen ist, spielte sie 1976 auf der Ostberliner Volksbühne mit Benno Besson, M. Karge und M. Langhoff. Nach ihren Auftritten auf der Berliner Volksbühne hat sie auch am Pariser, Bochumer, Berliner, Frankfurter und Münchner Theater gespielt. Sie hat in Filmen wie ,Happy Birthday Türke', ,Die Reise in die Nacht', ,Eine Liebe in Istanbul', ,Yasemin', ,Freddy Türkenkönig' und ,Airport, Rückflug nach Teheran' mitgespielt. Theaterstücke hat sie geschrieben und auch inszeniert. Özdamar lebt seit 1986 als freie Schriftstellerin in Berlin (vgl. Ekiz 2007: 39). Während Topçu (vgl. 2009: 705ff.) betont, dass Özdamar seit 1976 in Deutschland lebt, deshalb die deutsche Sprache gut beherrscht und in ihren Werken einen anderen Sprachstil pflegt als deutsche Schriftsteller, geht Aytaç (vgl. 1997: 1) davon aus, dass Özdamar ihre eigene Kultur in ihren Werken widerspiegelt. Milz (vgl. 2000: 4) beschreibt Özdamar als eine Schriftstellerin, die nicht nur ethnischkulturell, sondern gleichzeitig gesellschaftlich geschlechtsspezifischen Bezeichnungen widerspricht und einen ,Ausschluss von Inklusivität' erlebt. Laut Kocadoru (vgl. 2003: $35,66)$ zählen Özdamars Werke zur Literatur des heutigen Deutschland; indem sie ihre Themen aus der Türkei und aus Deutschland aussucht und diese mit einem anderen Deutsch ihrem Publikum vorstellt, verwirklicht sie eine interkulturelle Interaktion. Sağlam (vgl. 2006: 68) betrachtet Özdamar als eine Schriftstellerin, die in Deutschland lebt, Deutsch schreibt und das Problem ,Fremdheit' als Hauptthema behandelt. Sakall (2018: 19) dagegen stellt Özdamar als eine der Schriftsteller vor, die ,die Fremdheit überwunden haben und ihre Muttersprache mit der zweiten Sprache zusammen verwenden“ und somit einen neuen Erzählstil schaffen. Dank ihres neuen Stils hat Özdamar mit ihrem Werk ,Mutterzunge , in dem sie vom geteilten Deutschland und Berlin mit ihrer kontroversen Vergangenheit erzählt hat, als erste türkische Schriftstellerin den Ingeborg-Bachmann-Preis erhalten (vgl. Adelson 2002: 328). Dieser Literaturpreis ist ein Beweis, dass Özdamar eine weltweite Schriftstellerin ist. Dementsprechend sind mehrere Werke von Özdamar Thema weltweiter, wissenschaftlicher Untersuchungen geworden (Jankowsky 1997; Milz 2000; Johnson 2001; Lüling 2003; Brandt 2004; Göttsche 2006; Littler 2012). Insbesondere wurde der Roman ,Die Brücke vom Goldenen Horn' in der Türkei und im Ausland in mehreren wissenschaftlichen Arbeiten behandelt. Als eine der wichtigsten Arbeiten ist die von 
Gutjahr (vgl. 2007: 118-125) zu betrachten. Im Jahr 2007 veröffentlichte Gutjahr sein Buch ,Einführung in den Bildungsroman', in dem er neben Goethe, Hermann Hesse und Gottfried Keller auch Özdamars Roman ,Die Brücke vom Goldenen Horn` erwähnt. Krause (vgl. 2000: 84) betont in seiner Arbeit, dass Özdamar türkische Sprichwörter und Ausdrücke direkt ins Deutsche übersetzt und somit einen anderen Stil mit einer interkulturellen Perspektive verwendet. In Krauses Arbeit ist das Schattenmotiv interpretiert worden. Mecklenburg (vgl. 2004: 1-21) analysierte in seiner Arbeit ,die Entfremdung“ im Roman ,Die Brücke vom Goldenen Horn'. Außer dem Roman ,Die Brücke vom Goldenen Horn' untersuchte Mecklenburg auch die Romane ,Das Leben ist eine Karawanserei, hat zwei Türen, aus einer kam ich rein, aus der anderen ging ich raus. und ,Seltsame Sterne starren zur Erde' vor ihrem historischen und sozialen Hintergrund und stellte türkische und deutsche Strukturen heraus. Im Roman ,Die Brücke vom Goldenen Horn' schreibt er über lustige Motive und informiert über das türkische Frauenbild. Andererseits betont Theilen (vgl. 2006: 320) in seiner Arbeit, dass Özdamar ihren Ausgangspunkt im Roman ,Die Brücke vom Goldenen Horn' von nationaler Literatur zur universalen Literatur nimmt sowie mit unterschiedlichen Identitäten und deren Fakten in der Gesellschaft dem Roman einen dokumentarischen Effekt verleiht. Er bietet auch eine Analyse des historischen Hintergrunds der Einwanderungsliteratur. Boa (vgl. 2006: 530) lenkt in seiner Arbeit die Aufmerksamkeit darauf, dass Özdamar in ihren zwei Romanen (,Die Brücke vom Goldenen Horn ' und ,Das Leben ist eine Karawanserei, hat zwei Türen, aus einer kam ich rein, aus der anderen ging ich raus. ') autobiographische Züge verwendet hat und dass sie zwischen zwei Kulturen steht. Es gibt nicht nur Abschnitte des Lebens der Ich-Erzählerin, sondern es werden auch kulturelle Übertragungen von der Türkei und von Deutschland vollzogen. Wagner-Egelhaaf (vgl. 2009: 354) bringt in seiner Arbeit Özdamars Namen mit Goethe und Barthes zusammen. In Webers (vgl. 2010: 38) Arbeit wird untersucht, wie moslemische Frauen zum Arbeiten nach Deutschland gegangen sind und was sie der deutschen Gesellschaft gebracht haben. Dargestellt wird ebenfalls aus der Perspektive einer moslemischen Frau, welche Einstellung die moslemische Gesellschaft zur Sexualität hat. Er betont, dass Özdamar das Bild der türkischen Frau als Frau mit Kopftuch und als Putzfrau somit zerstört hat. Bayrak und Reininghaus (vgl. 2013: 12) untersuchten den Begriff ,Migration“ hinsichtlich von Raum und Raumlosigkeit in den Werken ,Selim oder die Gabe der Rede“ von Sten Nadolny und ,Die Brücke vom Goldenen Horn' von Özdamar. Marc Augés Theorie der Raumlosigkeit steht im Vordergrund dieser Kulturstudie, in der Kulturvergleiche beider Romane gemacht wurden. Das Hauptthema der Studie ist die Raumlosigkeit nach der Migration und in deren Folge auch Identitätslosigkeit. In diesem Teil der vorliegenden Arbeit wurden die Schriftstellerin, der theoretische Rahmen und verwandte Studien dargestellt. Im nächsten Teil der Arbeit wird die Methode erläutert.

\section{Methodik}

In diesem Teil der vorliegenden Arbeit sind Informationen über das Forschungsdesign und die Datenerhebungsmethode enthalten, die verwendet werden. 


\section{Forschungsdesign}

Mit der vorliegenden Arbeit wird bezweckt, aus dem Blickwinkel der Schriftstellerinnen, die eine Minderheit innerhalb der Migrantenliteratur bilden, das Frauenbild einer Frau darzustellen. Ein weiteres Ziel der Arbeit ist, neben den Frauenbildern der sogenannten Zeit auch die soziokulturelle Lage in Deutschland und in der Türkei darzustellen. Dementsprechend wurde der Roman ,Die Brücke vom Goldenen Horn` von Emine Sevgi Özdamar ausgewählt. Gemäß der Validität und Zuverlässigkeitskriterien der Studie wurden qualitative Daten verwendet. Die Arbeit wurde mit einem leserorientierten Ansatz zur Textanalyse analysiert, der einer der qualitativen Forschungstypen ist. Das Werk ist von zwei Forscherinnen durchgelesen und interpretiert worden, so dass die Themen des Werkes in den Vordergrund gebracht worden sind. Die hervorgebrachten Themen wurden untereinander verglichen und mit direkten Zitaten aus dem Werk unterstützt. Filizok (vgl. 2005: 12), der die Textanalyse nicht nur auf den Gedanken beschränkt, den der Autor dem Leser vermitteln möchte, sondern auch daran interessiert ist, wie der Autor mit dieser Idee umgeht, definiert die Textanalyse als ,Versuch, die Gedanken und Gefühle im Text zu erfassen und zu entdecken, wie der Autor diese Gedanken ausdrückt und wie er uns seine Emotionen durch die Möglichkeiten der Sprache vermittelt (Filizok 2005: 12)“". Im Werk werden die Ansicht, Gefühle und Gedanken der Frauen und zugleich die soziokulturellen Ereignisse in Deutschland und in der Türkei durch die Auffassung einer Frau vorgestellt. Bei der werkimmanenten Interpretation sind die Bilder der Ereignisse, die der Leser erhalten hat, thematisiert worden und mit direkten Zitaten aus dem Werk dargestellt worden.

\section{Datenanalyse}

Die Daten der Arbeit sind aus dem Roman namens ,Die Brücke vom Goldenen Horn ${ }^{\text {' der }}$ Schriftstellerin Emine Sevgi Özdamar erhoben. Der Roman ist von den Forscherinnen der vorliegenden Arbeit kritisch gelesen worden und dementsprechend wurden Motive von Frauenbild und -rollen aus dem Roman herausgearbeitet. Darüber hinaus wurden einige Informationen der Schriftstellerin über die historische und soziale Struktur in Deutschland und in der Türkei, die im Hintergrund angedeutet werden, zusammengestellt. Um zur Validität und Zuverlässigkeit der Studie in Übereinstimmung mit diesen festgelegten Themen und Hintergründen beitragen zu können, werden direkte Zitate aus dem Roman vorgestellt. Die Daten wurden durch eine werkimmanente Textanalyse erreicht. Die Werkimmanente Textanalyse oder die sogenannte Werkimmanente Interpretation ist eine methodische Richtung der Literaturwissenschaft. Laut Schlingmann (vgl. 1985: 91f.) bedeutet eine Werkimmanente Textanalyse, nicht ein literarisches Werk ohne biographische und historische Kenntnisse aus sich selbst heraus zu erklären, sondern der Nachweis soll manifestieren, dass und wie es in sich selber stimmt. 


\section{Ergebnisse}

Das Werk ,Die Brücke vom Goldenen Horn' von Emine Sevgi Özdamar besteht formal gesehen aus zwei Teilen. Der erste Teil des Romans heißt ,Der beleidigte Bahnhof ${ }^{\star}$, in diesem Teil erzählt Özdamar, wie sie mit 17 Jahren nach Deutschland ging und dort arbeitete, um eine Theaterausbildung machen zu können. Im zweiten Teil namens ,Die Brücke vom Goldenen Horn' beginnt die Geschichte in Istanbul und setzt sich anschließend mit Abschnitten aus Anatolien fort. Von dort geht es wieder nach Istanbul, bis die Hauptfigur in den Zug nach Deutschland steigt. Obwohl sich der Roman mit dem Leben der Schriftstellerin überschneidet, ist die Hauptfigur ohne Identität. Im ganzen Roman wird der Name der Hauptfigur nicht erwähnt. Die Schriftstellerin wollte vielleicht somit Verallgemeinerungen über das Leben mehrerer Frauen treffen, in dem sie sich selbst aber verbergen konnte. Bemerkenswert ist auch, dass außer der Hauptfigur alle anderen Figuren eine Identität haben. Ein weiteres bemerkenswertes Detail in diesem Roman ist, wie die Hauptfigur ihre Jungfräulichkeit verliert. Der Roman teilt dem Leser mit, wie man sich physisch und geistig ändert, wenn man die Jungfräulichkeit verliert. Und dabei reifer wird, den eigenen Blickwinkel ändert, stärker wird, sich für die Gesellschaft befleißigt und zum Wohle der Menschheit arbeiten muss. Im Vordergrund des Romans steht die Intertextualität. Özdamar nennt mehrmals die Autoren und Dichter, die sie gelesen hat und bietet auch Ausschnitte aus ihren Werken. Historische und soziale Hintergründe bringt sie in gut auswendig gelernten Schlagzeilen hervor. In diesen Schlagzeilen wird nicht nur über die Türkei und Deutschland berichtet, sondern auch die Weltpresse dieser Zeit erwähnt. Während die Schriftstellerin von den politischen Ereignissen an den türkischen Universitäten der 70er Jahre erzählt, stellt sie auch ihre eigene politische Haltung dar. Ihre sozialistische Haltung bringt sie dazu, nach Anatolien zu fahren, um den armen Bauern helfen zu können, sich besser zu äußern. Sie trägt das Leben der Bauern, das unter schwierigen Bedingungen stattfindet, in die Zeitungsartikel. Sie fühlt sich wegen der Armut der Menschen schuldig, weil sie aus einer bürgerlichen Familie stammt, weil sie keine finanziellen Probleme hat und erwähnt auch deshalb im Roman das Geld nicht so oft. Sie bemüht sich um arme Menschen, sie wird sogar wegen ihrer Meinungen in Haft genommen, aber wegen ihres Traums vom Theater vermeidet sie alle möglichen Schwierigkeiten. Und auch nur aus diesem Grund verlässt sie ihre eigene Heimat und kehrt nach Deutschland zurück.

Özdamar lässt im Roman nicht nur die Kultur, in der sie geboren und aufgewachsen ist widerspiegeln, sondern gibt auch Beispiele aus der deutschen Kultur, die ihr eigentlich fremd ist. Es gibt so manches in beiden Kulturen, was Özdamar nicht akzeptiert, worüber sie sich Gedanken macht, was ihr fremd ist. Somit erlebt auch Özdamar das Identitätsproblem, das das Hauptproblem der zweiten Generation war. Dieser Fakt ist im Roman von Anfang an zu bemerken. Das wichtigste Symbol der zweiten Generation wird mit der Brücke als Metapher dargestellt (vgl. Kocadoru 2004: 35), damit wird die Identitätsverwirrung und wie Özdamar zwischen zwei Ländern steckengeblieben ist gezeigt. Özdamar vergleicht sich mit der Brücke; mit einem Fuß in der Türkei und mit dem anderen in Deutschland, eine Doppelidentität (vgl. Cengiz 2010: 191). Die Verwirrung zwischen zwei Kulturen erlebt sie am meisten bei dem Thema 
Jungfräulichkeit. Mit anderen Worten war Sexualität das wichtigste Thema zwischen den Kulturen. Özdamars traditionelle türkische Identität ließ sie ständig daran erinnern, dass sie vor der Hochzeit mit keinem Mann Sex erleben sollte und dieses Gewissen war überall mit ihr, wie ein Schatten. Doch solange sie in Deutschland lebte, fühlte sie sich wie eine Deutsche, frei und weit entfernt von den Traditionen. Sie möchte mit dem Mann zu schlafen, der ihr gefällt, für den sie etwas empfindet. Auch anderen türkischen Frauen versucht sie aufzuerlegen, dass sie ihre Jungfräulichkeit aufgeben sollten. In dieser Krise der sexuellen Identität lernt sie sehr viele Männer kennen, bleibt aber immer unter dem Druck der türkischen Identität. Die Identität, die ihr sagt, dass sie nur eine gute Theaterschauspielerin werden kann, wenn sie ihre Jungfräulichkeit verliert und die Identität, die ihr sagt, sie soll sich bis zur Hochzeit schützen. Diesem Konflikt zwischen den zwei Identitäten kann man im Roman folgendermaßen begegnen:

\begin{abstract}
Unser kommunistischer Heimleiter hatte einen guten Freund, Ataman. Wenn wir aus dem Bus stiegen, streckte er uns seine Hände entgegen, damit wir im Schnee nicht ausrutschten, wir kicherten, nahmen aber nicht seine Hand. Dann lachte Ataman und sagte: "Sonst gehen eure Diamanten verloren, oder? Diamanten, Diamanten, Mädchen, gebt eure Diamanten her!« Immer wenn wir Ataman sahen, lachte er und sang: »Diamanten, Diamanten, wann gebt ihr eure Diamanten her?« Er rief auch: »Diamanten muss man wie Kleingeld ausgeben« (Özdamar 1998: 56).
\end{abstract}

Özdamar betont in ihrem Werk mehrmals das Thema Jungfräulichkeit, indem sie die Jungfräulichkeit als einen Diamanten beschreibt. Nach großen Kämpfen mit ihrem Gewissen nimmt sie sich vor ihren Diamanten loszuwerden. Diese Gefühle drückt Özdamar wie folgt aus;

\footnotetext{
Ich wollte endlich meinen Diamanten hergeben. Ich dachte, bevor ich nach Istanbul zurückkehre, muss ich mich in Berlin vor diesem Diamanten retten. Engel hatte ihren Diamanten hergegeben, Gutsio hatte keinen Diamanten mehr, oben in der sechsten Etage das Mädchen mit dem toten Embryo hatte auch keinen Diamanten. Und alle zogen genauso wie ich ihre Mäntel an und aus und konnten die Türe aufmachen. Briefe öffnen. Eine Zigarette rauchen. Ein Licht ausmachen. Makkaroni schmeckten ihnen weiter. Sie konnten sich im Kino auch ohne Diamant einen Film ansehen. Ich lag im Bett und schwor mir bei den an der Wand vorbeifahrenden Autoscheinwerfern, dass ich mich vor meinem Diamanten retten würde (ebd.: 117).
}

Im Werk ist deutlich zu sehen, dass Özdamar sehr oft ihre Meinung über die Jungfräulichkeit schildert. Sie ist zwischen den Gedanken steckengeblieben, die Jungfernhaut verlieren oder den Diamanten retten? (mehr dazu siehe ebd.: 52ff., 70 und 99). Özdamar, bringt auch den Kampf gegen ihr Gewissen zum Ausdruck, sie erklärt dem Leser mit folgenden Worten, dass sie ihre Jungfräulichkeit als übermäßig und unnötig ansieht, als etwas, dass sie loswerden muss: 
Ich saß im Kino und dachte nur, wie ich es schaffen könnte, mich heute Abend von meinem Diamanten zu befreien. Der Film war alt, er riss ein paarmal, und im Kino gingen kurz die Lichter an, bis man den Film wieder geklebt hatte. Auch die Tonspur war zerkratzt, aber ich hörte sowieso nicht dem Film, sondern nur den drohenden Sätzen in meinem Kopf zu: Du Nutte, wenn du dich nicht heute Abend von deinem Diamanten befreist, wirst du dich nie retten, dann wirst du als Jungfrau heiraten und dich als Jungfrau einem Mann verkaufen. Ich machte Pläne im Kopf, wie ich den hinkenden Sozialisten dazu bringen könnte, mich heute Abend von meinem Diamanten zu befreien (ebd.: 156).

Özdamar macht dem Leser in der ersten Hälfte deutlich, wie wichtig die Jungfräulichkeit in der türkischen Kultur ist. Obwohl Özdamar denkt, dass sie die Jungfräulichkeit loswerden muss, macht sie sich Gedanken darüber, dass ihr Leben sich verändern wird. In dem Roman, in dem eigentlich ihr eigenes Leben erzählt wird, wird ein Verfremdungseffekt benutzt. Die Erzählerin nimmt immer, wenn sie einem Mann näherkommt, auf einmal eine neutrale Erzählperspektive ein. Es ist bemerkenswert, dass die Ich-Erzählerin plötzlich zu einem personalen Erzähler wird. Özdamar erzählt von ihrem ersten Geschlechtsverkehr, den sie mit ihrer ersten Liebe Jordi hatte, aus der dritten Person Singular. Sie verhält sich so, als ob nicht sie mit Jordi zusammen sei, sondern jemand anders. Sie tut so, als ob sie ihre Jungfräulichkeit, die sie lange Zeit als Diamanten beschrieben hat, nicht mit Jordi verloren hätte. Aus diesem Grund ist Özdamar anders als die meisten türkischen Frauen der 70er Jahre, sie bricht das Tabu ,kein Sex vor der Hochzeit‘. Außerdem ist Özdamar von der ersten Frauengeneration zu unterscheiden, denn sie war nach Deutschland gegangen, um eine Theaterausbildung zu machen. In der ersten Generation war die Frau immer in Deutschland, damit sie viel Geld verdient, was meistens unter schlechten Bedingungen geschah, und dieses Geld musste sie ihrer Familie schicken. Hier nun eine Frau, die für eine Theaterausbildung nach Berlin kam und eine gute Theaterschauspielerin werden wollte. Diese junge Frau war sogar auch in anderen europäischen Ländern.

Im Roman zeigen sich mehrere Frauenbilder. Im ersten Teil des Romans wird nicht nur von Özdamar erzählt, sondern von mehreren Frauen, die ihre Familien in der Heimat zurückgelassen haben, um Geld zu verdienen, junge Mädchen, die zum Studieren gekommen sind, werden im ,Wonaym“ beschrieben. Özdamar hat diese Frauen meistens in zwei Gruppen geteilt:

\footnotetext{
Wenn unser kommunistischer Heimleiter mit einer Frau sprach, fing er seine Sätze immer mit dem Wort »Zucker« an. Wenn er zu mehreren Frauen sprach, sagte er »Zuckers«. »Zuckers, geht, setzt euch hin, ich komme gleich«, »Zucker, hier ist ein Brief für dich.« Die Frauen, die ihn liebten, fingen auch miteinander an, sich mit »Zucker« und »Zuckers« anzusprechen. Die Frauen, die ihn nicht liebten, sagten nicht »Zucker« zueinander. So teilte sich langsam das Frauenwonaym auf in die Frauen, die »Zucker» sagten, und in die Frauen, die nicht »Zucker« sagten. [...] Auch der Morgenbus, der uns zur Fabrik brachte, teilte sich in zwei Frauengruppen auf. Die Frauen, die nicht »Zucker« sagten, sondern »Esels, legt euch hin!«, setzten sich jetzt als Gruppe vorne in den Bus, und die, die »Zucker« sagten und Esels waren, hinten in den
} 
Bus. [...] So teilten sich langsam auch in der Fabrik die Frauen in die, die in der Toilette schliefen, und in die, die in der Toilette nicht schliefen (ebd.: 36f.).

Özdamar beschreibt die Frauen, die nach Deutschland kamen und dort in Fabriken wie Roboter arbeiteten mit folgenden Sätzen:

In der Arbeitshalle gab es nur Frauen. Jede saß da allein vor einem grüngefärbten Eisentisch. Jedes Gesicht schaute auf den Rücken der anderen. Während man arbeitete, vergaß man die Gesichter der anderen Frauen. Man sah nur Haare, schöne Haare, müde Haare, alte Haare, junge Haare, gekämmte Haare, ausfallende Haare (ebd.: 25f.).

Das Frauenbild in Özdamars Werk beschreibt die Frau, die zum Arbeiten nach Deutschland geht, dieses Bild ähnelt den Bildern von Bekir Yıldı (1995) in den Werken ,Sahipsizler ${ }^{6}$ und ,Motorize Köleler'. Während Bekir Yıldız von Männern erzählt, die wie Roboter ständig arbeiten, erzählt Özdamar von Frauen, die genauso hart arbeiten. Frauen, die sich während der Arbeit nicht ins Gesicht schauen, werden wie Roboter dargestellt. Der einzige Unterschied zwischen den arbeitenden Frauen sind ihre Haare. Wie auch im obigen Zitat zu erkennen ist, hat Özdamar mit diesen verschiedenen Haaren unterschiedliche Frauenbilder geschildert. Nämlich junge, ältere, gepflegte, ungepflegte, kummervolle Frauen, die in Deutschland arbeiten mussten.

Im zweiten Teil des Romans ist die Schriftstellerin zurück in der Türkei und beschreibt dem Leser nun verschiedene Frauenbilder aus der Türkei. Im Teil ,Stimmen der Mütter' stellt Özdamar das Mutterbild durch ihre eigene Mutter dar.

\footnotetext{
Tante Topus kam und sagte: »Schämst du dich nicht vor Allah? Du hast deine Mutter krank gemacht.« - »Weißt du, wie viele Menschen jetzt vor Hunger sterben? « fragte ich Tante Topus. »Wirst du denn die Welt retten?« - »Ja«, sagte ich, »ich will die Welt retten.« - »Wenn du die Welt retten willst, warum machst du deine Mutter krank? Ist sie nicht auch von dieser Welt?« (ebd.: 282).
}

Meine Mutter sah die leere Wohnung, ich sagte: »Es war eine Kleinbürgerwohnung.« »Ich weine für dich, weil ich weiß, dass du später sehr bereuen wirst, was du getan hast« sagte sie (ebd.: $315 f$.).

Özdamar war dagegen, dass das arme Volk, das sie in Anatolien erlebt hatte, so viele Kinder zur Welt bringt. Auch sie selbst hatte zwei Abtreibungen hinter sich. Ihrer Meinung nach war es sinnlos, Kinder auf diese Welt zu bringen,

Ich wurde wieder schwanger, aber wir sagten: «Kein Kind für diese kaputte Gesellschaft« (ebd.: 295). 
Mit Hilfe einer idealistischen Ärztin vermittelt die Schriftstellerin in dem Roman, wie oft und warum die Frauen in Dörfern so viel gebären:

Im Dorfgemeinderaum hatte eine Ärztin um sich die Bäuerinnen versammelt und wollte ihnen allen eine Spirale als Verhütungsmittel einsetzen. Die Bäuerinnen genierten sich und lachten. Die Ärztin sagte: »Die Welt geht zum Mond, und ihr macht so viele Kinder wie die Hühner, und kein Küken bekommt etwas Richtiges zu essen« (ebd.: 261).

In der türkischen Gesellschaft hat der Mann das Wort, die Frauen gehorchen ihren Männern, patriarchalisch geprägte Frauen in Anatolien werden in den folgenden Sätzen von Bäuerinnen dargestellt:

Die Bäuerinnen sagten: »Lass noch einen Sommer vorbeigehen, wir müssen erst unsere Männer fragen« (ebd.: 261)

Den damaligen Sitten in Anatolien entsprechend wurden viele Frauen, deren Mann verstorben ist, dazu gezwungen ihren Schwager zu heiraten. Im Folgenden drückt Özdamar aus, wie sie mehrmals verheiratete Frauen mit berühmten Schauspielerinnen vergleicht:

Frauen, die viele Männer hatten, erinnerten mich an die Hollywoodschauspielerinnen Zsa Zsa Gabor, Liz Taylor und an türkische Bäuerinnen. Deren Ehemänner gingen in die Großstadt arbeiten, arbeiteten dort als Lastträger oder Bauarbeiter, schliefen auf der Baustelle, starben jung oder starben im Krieg, und ihre Frauen gab man ihren Brüdern zur Frau. So hatten die türkischen Frauen aus den Dörfern auch viele Ehemänner (ebd.: 91).

Özdamar betont, dass das Leben der anatolischen Frau schwierig genug war und dazu kam noch, dass die trauernde Hinterbliebene ihren Schwager heiraten musste. Die anatolische Frau steht auch in Deutschland unter Druck, auch wenn ihr Mann nicht dabei ist. Davon erzählen die Frauen im Wonaym:

Ich übte meine Sätze im Bus, und abends im Wonaym merkte ich, dass auch die anderen Frauen irgendetwas übten. Eine übte, wie sie ihren Weg aus dem Stadtzentrum zurückfindet. Sie ging dort öfter in die Kaufhäuser und hatte eines Abends ihren Weg verloren, weil im Berliner Winter die Dunkelheit sehr schnell herunterkam. Zuerst hatte sie geweint, dann sagte sie zu sich, um sich zu beruhigen: »Gut, dass mich zu Hause kein Mann erwartet. [...] Eine andere Frau übte, auf einer Rolltreppe rückwärts zu laufen. Auch sie sagte: »Gut, dass ich keinen Mann habe, wenn er mich so sehen würde, würde er mir meine Haare ausreißen.« Jede Geschichte endete mit einem Mann. [...] Die eine Gruppe der Frauen sagte: »Gut, dass wir keinen, Mann haben ...«, die anderen sagten: »Leider haben wir - keinen Mann ...« Aber jeder Satz, egal, ob er mit »gut« oder 
»leider« anfing, gebar immer einen Mann. Das Wort »Mann« war wie ein großer Kaugummi, den sie gemeinsam kauten (ebd.: 64f.).

Türkische Männer in Deutschland waren dermaßen an die patriarchische Gesellschaft gewohnt, dass sie sich bei alleinstehenden türkischen Frauen in einige Dinge einmischten. Dieses Gefühl war den Frauen von der Türkei bekannt, aber jetzt taten es fremde türkische Männer in Deutschland. Özdamar erwähnt diese Situation wie folgt:

\begin{abstract}
Obwohl die alleinstehenden Frauen schon länger als ein Jahr in diesem Wohnheim wohnten, fingen die Ehepaare sofort an, sich um die Ehre der Frauen im vierten, fünften und sechsten Stock zu kümmern. Manche der alleinstehenden Frauen hatten nach ihrer Meinung ihre türkische Ehre in Berlin wie ein Kleid ausgezogen, und besonders die Männer wollten ihnen dieses Kleid wieder anziehen (ebd.: 109).
\end{abstract}

Özdamar beschreibt in ihrem Roman mehrere Frauenbilder. Eines dieser Bilder ist die Frau, die aufgrund von Armut ein schwieriges Leben hat und deshalb auf den Strich gehen muss:

»Woher kommst du, meine Schöne, so spät in der Nacht?« Gut, sollen sie doch denken, dass ich eine Hure bin, dachte ich plötzlich. Huren hatten auch Mütter gehabt wie ich. Und ein Mädchen, das richtig arm war, konnte sich innerhalb von zwei Tagen in der Hurenwelt wiederfinden. Nur zwei Tage Hunger, zwei Tage ohne Bleibe und keine zwei Menschen, die ihr helfen konnten, das reichte. Dann klopfte ein Mädchen an die Tür eines Herrenhauses (ebd.: 209f.).

Özdamar teilt in ihrem Roman mit, was sie alles über Prostituierte erfahren hat. Beim Vergleich von Prostituierten in der Türkei und in Deutschland bringt sie den kulturellen Unterschied folgendermaßen in den Vordergrund:

Unten auf der Straße standen Huren. Es regnete, und sie stellten sich in die Haustüren. Wenn eine Hure einen zu großen Busen hatte, stand ihr Busen etwas vor, und der Regen fiel zwischen ihre Brüste. Die älteren Huren, sogar 60-70jährige Frauen, trugen Hüte und zu viel Lippenstift. [...] Bremsende Autoreifen spritzten den Regen auf die Miniröcke der Huren. Manchmal hatte eine Hure einen kleinen Hund. Auch diese Hunde waren nass vom Regen, schüttelten ständig ihre Köpfe und Körper und machten mit dem Regenwasser die Beine der Huren nass. Rezzan und Gül wiederum machten die Huren vom Fenster aus nass. Sie schütteten ein Glas Wasser über die Huren und zogen sich danach schnell ins enge Zimmer zurück. Dann saßen sie im Bett, lachten und gossen nach einer halben Stunde noch einmal ein Glas Wasser über eine Hure und über ihren Hund. Die Huren schimpften, ihre Hunde bellten. An einer Bushaltestelle fuhr gerade ein Bus ab. Dann kam ein Mann, wartete auf den nächsten Bus, während eine Hure mit ihrem Kunden aus dem Hotel herauskam (ebd.: 87). 
Im Gegensatz zu den Prostituierten in Deutschland, schildert sie die in der Türkei wie folgt:

Die alten Huren saßen an einem Ofen, die jungen erzählten. Es war kalt, deswegen trugen viele Huren kurze Socken aus Wolle und Wollwesten. Jede Hure hatte zwei Betten, eins war ihr eigenes Bett, das Bett einer Prinzessin, groß, mit einer schönen Bettdecke, das andere war ihr Arbeitsplatz (ebd.: 288).

Neben diesen unterschiedlichen Frauenbildern im Roman, wird auch dargestellt, wie ein junges Mädchen das nach Deutschland geht, zwischen zwei Kulturen stecken bleibt und u.a. einen Kulturschock erlebt. In den folgenden Zitaten ist diese Situation deutlich zu sehen:

Ich hatte in Berlin noch keinen deutschen Offizier oder Soldaten gesehen. Die einzigen Uniformen, die ich gesehen hatte, waren weiße oder graue Kittel in der Fabrik (ebd.:

71f.).

Ich lief einmal mit einer türkischen Studentin zum Café Steinplatz und hatte meinen Arm in ihrem Arm. Sie sagte zu mir: »Nimm deinen Arm weg, sonst werden sie glauben, dass wir lesbisch sind." Ich war früher in Istanbul mit Frauen immer Arm in Arm gelaufen, aber niemand dachte dabei an Lesben. Auch Männer liefen dort Arm in Arm (ebd.: 154).

Zur selben Zeit, in der die Schriftstellerin Kulturschocks in Deutschland erlebte, gab es in der Türkei ein großes Interesse an Europa. Europa gesehen und erlebt zu haben, eine Ware aus Europa zu besitzen, all dies wurde als etwas Besonderes empfunden. Die Bewunderung der türkischen Gesellschaft gegenüber Europa wird im Folgenden dargestellt:

Ich saß neben meinem Vater, und er sagte zu einer Frau, die hinten saß: »Das ist meine Tochter, sie kommt gerade aus Deutschland, sie hat Europa gesehen.« Die Frau antwortete: »Europa gesehen zu haben ist eine feine Sache. Man sieht einem Menschen im Gesicht an, dass er Europa gesehen hat. Die Europäer sind fortschrittlich, wir treten mit unseren Füßen auf der Stelle und bewegen uns einen Schritt vor und zwei Schritte zurück« (ebd.: 103).

Europäisches Aspirin heilte Herzkrankheiten. Bei europäischen Stoffen konnte man aus 40 Metern Entfernung erkennen, wie gut sie waren. Europäische Schuhe konnten nie kaputtgehen. Europäische Hunde hatten alle in den europäischen Hundeschulen studiert. Europäische Frauen waren echte Blondinen. Europäische Autos machen keine Unfälle (ebd.: 242). 
Özdamar beschreibt dem Leser im Roman neben verschiedenen Frauenbildern, Kulturschocks und Bewunderung für Europa auch wichtige historische und soziale Ereignisse der Zeit in Deutschland und in der Türkei. Der Roman gleicht einem historischen Werk, indem es über Historisches und Soziales informiert. Im Folgenden einige Zitate, in denen dies der Fall ist:

In den Zeitungen gab es oft Fotos von dem regierenden Parteichef Demirel, der z.B. neben dem amerikanischen Präsidenten Johnson stand, sie schwitzten nicht (ebd.: 199).

Zwischen Asien und Europa gab es damals, 1967, noch keine Brücke (ebd.: 215).

Neben politischen Nachrichten aus der Türkei wird dem Leser auch mitgeteilt, wie das Leben dort damals aus sozialer Hinsicht zu betrachten war.

Die Armut lief wie eine ansteckende Krankheit durch die Straßen. Ich schaute auf die Armen wie auf Pestkranke und konnte nichts für sie tun (ebd.: 222).

Vieles wird im Roman ineinander verwoben erzählt, das alltägliche Leben der Erzählerin und politische Ereignisse der Zeit:

Wir standen vor dem dritten Treppeneingang und machten das Licht an. Er schob meine Bluse hoch, die Bluse mit den gelben Blumen, die ich getragen hatte als der Berliner Student Benno Ohnesorg beim Schach-Besuch in Berlin getötet worden war (ebd.: 227).

Die obigen Zitate und die folgenden Zitate aus dem Werk zeigen, wie unmittelbar die politischen Nachrichten im Roman in den Vordergrund gestellt werden:

De Gaulle will in die Türkei kommen. Die türkische Regierung ließ für de Gaulles Länge ein Bett bauen (ebd.: 248).

Im Roman von Özdamar werden mehrere historische und soziale Hintergründe auch indirekt beschrieben. Dementsprechend sind nur wenige direkte Zitate aus dem Roman hervorzuheben. Auch die Armut des anatolischen Volkes und ihre schwierigen Lebensbedingungen werden im Roman dargestellt:

In der Stadt [Hakkari] gingen wir für unsere Reportage auf die Suche nach Bauern, die drei Tage lang zu Fuß aus den verhungernden Dörfern hierhergelaufen waren, um Mehl zu kaufen. Einen fanden wir, er war dünn wie eine Nadel, seine Augen lagen tief in seinem Gesicht. Auf seinem Rücken trug er einen schweren Sack Mehl, er wollte sich gerade zu dem verhungernden Dorf auf den Weg machen. Als wir mit ihm sprachen, 
sahen wir hinter einem Gebüsch wieder unsere sechs Zivilpolizisten. Der Bauer sagte: »Wir haben die Blätter von den Bäumen gegessen wie die Tiere, aber jetzt sind auch keine Blätter mehr da. Wir sind tot, meine Tochter. Keiner gibt uns eine Hand. Wir haben in dieser blinden Welt den Jüngsten Tag gesehen. Die Kinder sind gestorben wie Blüten, die der Wind von den Zweigen weht. Sagt dem Staat, er soll uns aus den Helikoptern Gift herunterwerfen. Wir werden es essen und dann alle sterben. Das ist mein Wunsch vom Staat. Schreib das, meine Tochter« (ebd.: 271).

Mit direkten und indirekten Zitaten aus dem Roman ,Die Brücke vom Goldenen Horn “ von Özdamar wurde versucht, verschiedene Frauenbilder darzustellen. Es wurde versucht hervorzuheben, aus welcher Blickweise die türkische Gesellschaft der Frau gegenüber handelt. Auch unterschiedliche Kulturen und dementsprechende Kulturschocks sowie historische und soziale Ereignisse in Deutschland und in der Türkei wurden mit Zitaten aus dem Roman dargestellt. Am Ende der vorliegenden Arbeit wird eine allgemeine Bewertung vorgenommen.

\section{Schlussfolgerung}

In dieser Arbeit wurde versucht, in dem Roman ,Die Brücke vom Goldenen Horn 'von Özdamar, die eine Schriftstellerin der Migrantenliteratur ist und deren Romane autobiographische Züge aufweisen, unterschiedliche Frauenbilder herauszustellen. Neben den Frauenbildern im Roman spielt auch die Bereitstellung von Informationen für den Leser eine wichtige Rolle. Diese Informationen werden mit einer neutralen Perspektive der Schriftstellerin übermittelt. Sie steht den sozialen und historischen Ereignissen und Fakten dieser Jahre empfindungslos gegenüber. Weil ihre Herangehensweise an historische und soziale Ereignisse neutral ist, bietet Özdamar ihren Lesern Kenntnisse wie aus einem historischen Roman. Es treten mehrere Frauenfiguren und -bilder im Roman auf. Kurz zusammengefasst könnte man die aufgetretenen Frauenbilder folgendermaßen darstellen: Frauen aus Anatolien, die unter schwierigen Lebensbedingungen leben, mehrere Kinder zur Welt bringen, Frauen die ihren Schwager heiraten müssen, wenn der Ehemann stirbt und Frauen in patriarchalischen Gesellschaften. Migrantinnen, die den Druck ihres Mannes, auch wenn sie allein in Deutschland sind, spüren, wo dann noch türkische Männer in Deutschland sich zu Ehrenwächtern dieser Frauen erklären und die Frauen, die dazu gezwungen sind, wie mechanische Sklavinnen Geld in ihre Heimat zu schicken. Prostituierte, die unter schwierige Lebensbedingungen gedrängt werden und als andere Seite der Gesellschaft gesehen werden. Frauen, die sich um ihre Kinder Sorgen machen, ihretwillen auf sie verzichten. Frauen, die für ihre Träume endlos arbeiten und sogar ihre Heimat verlassen. Frauen, die für arme Menschen und Menschen unter schwierigen Bedingungen argumentieren, dass alle Menschen das Recht dazu haben unter guten Bedingungen leben $\mathrm{zu}$ können. Und sich aufgrund ihrer eigenen besseren Lebensbedingungen schlecht fühlende Frauen. All diese Frauenbilder werden dem Leser dargestellt. Während Özdamar Spuren dieser Frauen beschreibt, wird den Lesern auch von den historischen und sozialen Fakten dieser Zeit in Deutschland und in der Türkei erzählt. Der damalige Präsident, wichtige Ereignisse in der Türkei und auf der Welt werden mit 
Hilfe von Zeitungsüberschriften neutral dargestellt. Darüber hinaus erwähnt Özdamar die intensive Armut in Anatolien, die Frauen, die viele Kinder zur Welt bringen, und deren Kinder unter Hunger leiden. Auch von der Bewunderung gegenüber Europa in dieser Zeit wird erzählt, und wie vertrauensvoll die türkischen Menschen europäischen Produkten gegenüber waren. Im Endeffekt hat Özdamar neben ihrer schriftstellerischen Seite auch eine sehr gute Betrachterseite, die sie ihre Leser mitspüren lässt.

\section{Literaturverzeichnis}

Adelson, Leslie A. (2002): The Turkish Turn in Contemporary German Literature and Memory Work. The Germanic Review: Literature, Culture, Theory 77. 326-338.

Aksan, Doğan (2005): Şiir Dili. Dil ve Edebiyat Dergisi 1. 1-13.

Asutay, Hikmet/ Çarıkçı, Tuğba (2015): Göçün Ellinci Yılında Almanya'da Yükselen Değer: TürkAlman Göçmen Yazını. Humanitas-Uluslararası Sosyal Bilimler Dergisi 5. 17-32.

Aytaç, Gürsel (1997): Sprache als Spiegel der Kultur. Zu Emine Sevgi Özdamars Roman Das Leben ist eine Karawanserei. Mary Howard (Hg): Interkulturelle Konfigurationen. Zur deutschsprachigen Erzählliteratur von Autoren nichtdeutscher Herkunft. München: Iudicium Verlag.

Balcı, Umut (2010): Transkulturelle Dimensionen der Deutschsprachigen Literatur Türkischer Migranten und ihre Vermittlung im DaF-Unterricht. Doktorarbeit. Adana Çukurova-Universität Sozialwissenschaftliches Institut.

Balcı, Umut/ Akgün, Medeni (2018): Emine Sevgi Özdamar'1n Mutterzunge Adlı Eserindeki Kalıp İfadeler ve Türkçe Çevirisindeki Karşılıkları. Social Sciences Studies Journal 4. 2097-2103.

Bayrak, Deniz/ Reininghaus, Sarah (2013): Die Darstellung türkischer Migration zwischen Ort und Nicht-Ort. Züge und Bahnhöfe in Sten Nadolnys Selim oder Die Gabe der Rede und Emine Sevgi Özdamars Die Brücke vom Goldenen Horn. Alman Dili ve Edebiyatı Dergisi 29. 11-33.

Boa, Elizabeth (2006): Özdamar's Autobiographical Fictions: Trans-National Identity and Literary Form. German Life and Letters 59. 526-539.

Brandt, Bettina (2004): Collecting Childhood Memories of the Future: Arabic as Mediator Between Turkish and German in Emine Sevgi Özdamar's Mutterzunge, The Germanic Review: Literature, Culture, Theory 79. 295-315.

Can, Özber (2011): Göçmen Yazın Süreci Çerçevesinde Feridun Zaimoğlu ve Anlatısı. Selçuk Üniversitesi Edebiyat Fakültesi Dergisi (SEFAD) 25. 139-156.

Cengiz, Semran (2010): Göç, Kimlik ve Edebiyat. Zeitschrift für die Welt der Türken/ Journal of World of Turks 2. 185-193.

Çakır, Mustafa (2012): Türklerin Alman Kültüründeki Imajı. Hg. v. Zehra Gülmüş. Türk Alman İşgücü Anlaşması'nın 50. Yılında Almanya Türkleri. Eskişehir: T.C. Anadolu Üniversitesi Yayınları.

Ekiz, Tevfik (2007): Avrupa Türk Edebiyatı ve Bir Temsilcisi: Emine Sevgi Özdamar. Çankaya University Journal of Arts and Sciences 1. 33-47.

Er, Mutlu (2013): Yüksel Pazarkaya'nın 'Mediha' Adlı Eserinde Türk Kadını İmgesi. Diyalog Interkulturelle Zeitschrift Für Germanistik 1. 24-31.

Filizok, Rıza (2005): Metin Analizi ve Mehmet Kaplan. Ege Üniversitesi ders notlar1.

Ghaussy, Sohelia (1999): Das Vaterland verlassen. Nomadic Language and ,Feminine Writing‘ in Emine Sevgi Özdamar's „Das Leben ist eine Karawanserei“. The German Quarterly 72. 1-16. 
Göttsche, Dirk (2006): Emine Sevgi Özdamars Erzählung Der Hof im Spiegel: Spielräume einer postkolonialen Lektüre deutsch-türkischer Literatur. German Life and Letters, 59. 515-525.

Gutjahr, Ortrud (2007): Einführung in den Bildungsroman. Darmstadt: Wissenschaftliche Buchgesellschaft.

Hammond, Andrew (Hg.) (2016): The European Theme in Literature. The Novel and Europe: Imagining the Continent in Post - 1945 Fiction. London: Palgrave Macmillan.

Herzog, Andrea (2010): Transkulturelle Elemente bei Emine Sevgi Özdamar in „Die Brücke vom Goldenen Horn “ und „Mutterzunge“. Diplomarbeit. Universität Wien.

İlkılıç, Süreyya (2000): Das Deutschen- und Deutschlandbild in der türkischen Migrantenliteratur und eine sprachliche Untersuchung am Beispiel ausgewählter Texte von Aras Ören: „Bitte nix Polizei und „Berlin Savignyplatz“. Magisterprüfung der Neuphilologischen Fakultät an der EberhardKarls-Universität Tübingen.

Jankowsky, Karen (1997): “'German' Literature Contested: The 1991 Ingeborg-Bachmann-Prize Debate, 'Cultural Diversity', and Emine Sevgi Özdamar”. The German Quarterly 70. 261-276.

Johnson, Sheila (2001): Transnational Johnson, S. Ästhetik des türkischen Alltags: Emine Sevgi Özdamar's Das Leben ist eine Karawanserei. The German Quarterly. 74. 37-57.

Kocadoru, Yüksel (2003): Geçmişten Günümüze Almanya'da Almanca Yazan Türkler ve Emine Sevgi Özdamar, Eskişehir: Rema Matbaacılık.

Kocadoru, Yüksel (2004): Die dritte Generation von türkischen Autoren in Deutschland - neue Wege, neue Themen. Manfred Durzak / Nilüfer Kuruyazıc1 (Hg): Die andere Deutsche Literatur. Istanbuler Vorträge. Würzburg: Königshausen \& Neumann Verlag. 134-139.

Krause, Frank (2000): Shadow Motifs in Emine Sevgi Özdamar's Die Brücke vom Goldenen Horn: A Corrective to the Limitations of Current Debates on Inter-Cultural Issues. Debatte: Journal of Contemporary Central and Eastern Europe 8. 71-86.

Kuruyazıcı, Nilüfer (1996): Emine Sevgi Özdamars ,Das Leben ist eine Karawanserei‘ im Prozess der interkulturellen Kommunikation. Gesellschaft für interkulturelle Germanistik, Kongressakten. München: Iudicium Verlag. 179-188.

Kuruyazıcı, Nilüfer (1997): Der literarische Text als Kulturvermittler. Sevgi Özdamars Roman ,Das Leben ist eine Karawanserei'. Mary Howard Interkulturelle Konfigrationen: Zur deutschsprachigen Erzählliteratur von Autoren nichtdeutscher Herkunft. München: Iudicium Verlag. 635-643.

Littler, Margaret (2012): The Fall of the Wall as Nonevent in Works by Emine Sevgi Özdamar and Zafer Şenocak. New German Critique 39. 47-62.

Lüling, Aynur (2003): Frauenfiguren in türkischer Migrantenliteratur. Magisterarbeit. Hamburg: Diplomica Verlag.

Mecklenburg, Norbert (2004): Ein weiblicher Schelmenroman. Das Erzählprinzip der komischen Verfremdung in Emine Sevgi Özdamars Brücke vom Goldenen Horn. Studien zur deutschen Sprache und Literatur 16. 1-21.

Milz, Sabine (2000): Comparative Cultural Studies and Ethnic Minority Writing Today: The Hybridities of Marlene Nourbese Philip and Emine Sevgi Özdamar. Comparative Literature and Culture 2. 114.

Mutlu, Betül (2012): Divan Şiirinde Deniz İmgesi ve Şiir Öğretiminde Kullanılması. Doktora Tezi. Dokuz Eylül Üniversitesi Eğitim Bilimleri Enstitüsü. 
Oraliş, Meral (2001): Gurbeti Vatan Edenler. Gurbeti Vatan Edenler. Almanca Yazan Almanyall Türkler. Hg. v. Mahmut Karakuş/ Nilüfer Kuruyazıcı. Ankara: Kültür Bakanlığı Yayınları, S. 3550.

Özdamar, Emine S. (1998): Die Brücke vom Goldenen Horn. Köln: Kiepenheuer\&Witsch Verlag.

Öztürk, Kadriye (1999): Das Frauenbild in den Werken der deutschschreibenden türkischen Autorinnen (Vol.1112). TC Anadolu Üniversitesi Sosyal Bilimler Enstitüsü.

Sağlam, Fatma (2006): Renan Demirkan'ın Üç Şekerli Demli Çay Adlı Eserinde Yer Alan Türk ve Alman İmgelerine Karşılaştırmalı Bir Bakış. Milli Folklor 72. 58-70.

Sakallı, Cemal (2018): Göçmen Edebiyatı: “Ara Dilde” Yazmak. Monograf Edebiyat Eleştirisi Dergisi 9. 10-27.

Schlingmann, Carsten (1985): Methoden der Interpretation. Ditzingen: Reclam Verlag.

Theilen, Ines (2006): Von der nationalen zur globalen Literatur. Eine Lese-Bewegung durch die Romane Die Brücke vom goldenen Horn von Emine Sevgi Özdamar und Café Nostalgia von Zoé Valdés. Arcadia 40. 318-337.

Topçu, Hayrunisa (2009): Avrupa ve Amerika'da Türk Edebiyatı. Turkish Studies International Periodical for the Languages, Literature and History of Turkish or Turkic 4. 701-734.

Uysal-Ünalan, Saniye (2016): „Andere Räume“ und Europa-Bilder in Emine Sevgi Özdamars „Die Brücke vom Goldenen Horn“. XIII. Internationaler Türkischer Germanistik-Kongress an der Akdeniz Universität in Antalya. "Zukunftsperspektiven der Germanistik", Antalya, Turkey.

Wagner-Egelhaaf, Martina (2009): Autofiktion oder: Autobiographie nach der Autobiographie: Goethe-Barthes-Özdamar. Hg. v., Ulrich Breuer/ Beatrice Sandberg. Autobiographisches Schreiben in der deutschsprachigen Gegenwartsliteratur. Grenzen der Identität und der Fiktionalität. Band 1. München: Iudicium Verlag.

Weber, Beverly M. (2010): Work, Sex, and Socialism: Reading Beyond Cultural Hybridity in Emine Sevgi Özdamar's Die Brücke vom Goldenen Horn. German Life and Letters 63. 37-53.

Yıldız, Bekir (1995): Sahipsizler. İstanbul: Cem Yayınevi Yayınları. 